\title{
The importance of symmetry and complexity in the evaluation of complexity, interest and pleasingness
}

HY DAY

UNIVERSITY OF TORONTO

The Ss were required to rate on scales of subjective complexity and affect two sets of figures, one comprising a series of random polygons varying in number of sides from four to 160 , and the second, four series of symmetrical polygons varying in number of sides from 10 to 90 . Symmetry was found to be a significant determinant of rated complexity and affect, but did not affect these responses in the same way as did complexity.

A rapidly increasing number of studies have been concerned with the visual presentation of a series of figures varying along a continuum of objectively determined complexity while measuring verbal responses of subjective complexity and affect level (cf. Day, 1967; Munsinger \& Kessen, 1964; Thomas, 1966). The factor of symmetry has been explored less frequently (cf. Eisenman \& Rappaport, 1967) and much less rigorously. Barron \& Welsh (1952), for example, developed a preference scale whose items differed along both the complexity and a symmetry dimension, but later publications by Barron (e.g., 1963) referred to the scale as if it measured only complexity. Berlyne also used symmetry as one of the defining characteristics of complexity, together with amount of material, redundancy of parts, etc., when he constructed a set of figures made up of pairs, the members of which were purported to differ in level of complexity (Berlyne, 1958, 1963).

Following Attneave's definition of symmetry as a form of redundancy which reduces informational content in a visual display (Attneave, 1955), Munsinger \& Kessen (1964) tried to distinguish between number of turns in a random polygon and number of independent turns. They argued that symmetrical figures contain approximately half the number of independent turns compared with asymmetrical figures with equal numbers of total turns and thus half the uncertainty. They also argue that Ss make more highly affective responses to variations in uncertainty than to variations in complexity.

Recently, Day reported on a series of studies which examined subjective evaluations of complexity, pleasingness, and interestingness of random asymmetrical polygons (Day, 1967). He showed that subjective complexity increases monotonically with the number of sides; in the figures (objective complexity) interestingness describes an inverted $U$-shaped function over objective complexity, while pleasingness appears to vary randomly but generally tends to decrease with increasing objective complexity.

If symmetry is a factor within complexity, then symmetrical polygons should be rated subjectively less complex than asymmetrical polygons with equal numbers of sides, and ratings of interestingness and pleasingness should be equally and correspondingly affected. If, however, symmetry is a dimension of "figural goodness" but not of complexity per se, one should expect that affective ratings be altered but not necessarily in direct relation to subjective ratings of complexity.

\section{Stimulus Material}

The figures used in this study were of two kinds. One was a series of 22 random black-on-white solid polygons differing in number of sides from 4 to 160 , used in a previous study by this author (Day, 1967). The other was a set of symmetrical polygons constructed from eight of these figures $(10,14,20,28,40,54,70$, and 90 sided figures). Symmetrical shapes were made by cutting a print of one of the random figures into two equal sided halves and generating a mirror image from each half. Thus two symmetrical figures were generated, with the same number of sides as the original figure (but half the number of independent turns). Slides were then made of the figures with both horizontal and vertical symmetrical axes creating a total of four symmetrical shapes, two horizontal and two vertical from each of the eight figures.

Subjects

The Ss in this study were 348 students at a local teachers' college. They all participated in one group session in the auditorium of the school.

Procedure

Sixty figures were projected by means of a Kodak Carousel projector at $5 \mathrm{sec}$ exposures. Six practice slides, similar to the rest, were presented but were not scored. The other 54 figures were made up of 22 asymmetrical and 32 symmetrical figures in randomized order.

The Ss were required to rate the figures along 7point scales of complexity, pleasingness, or interestingness. Answer booklets were distributed so that Ss sat between others using different response indices. Resulis

Figure 1 shows the distribution of ratings of the three response scales. Subjective complexity appears to have reached a ceiling and leveled off; otherwise 


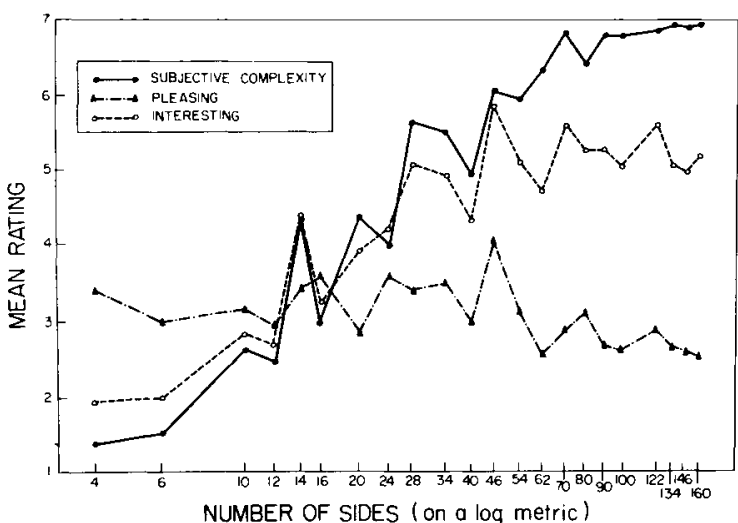

Fig. 1. Subjective evaluations of random polygons differing in number of sides.

the differences may have been greater. An analysis of variance reveals a significant complexity effect $(F=$ 183.62, $\mathrm{df}=2 / 345, \mathrm{p}<.001$ ), a significant response scale effect $(F=211.99, \mathrm{df}=21 / 7245, \mathrm{p}<.001)$, and a significant interaction effect $(F=83.97, \mathrm{df}=42 / 7245, \mathrm{p}<$ $.001)$. A trend analysis shows that linear and quadratic components are highly significant $(p<.001)$ for all three response scales, while the cubic component is significant $(F=4.31)$ at the $5 \%$ level for pleasingness only.

A comparison of trends shows that the linear components are significantly different for the three response scales ( $F=1564.43, p<.001)$ as are the quadratic components $(F=83.55, p<.001)$. While the quadratic component for the response scale of subjective complexity may merely be an artifact of the experimental design, and a function of the limits imposed by the range of the rating scales, the quadratic component of the interestingness scale has been found on a number of previous occasions and has been described as an inverted U-shaped function of interest over complexity (cf. Day, 1967). No significant differences were found for the cubic components ( $F=1.63$ ).

Correlations for the means of all three response indices with objectively defined complexity were found to be highly significant (complexity by subjective complexity, $r=.81$; complexity by interestingness, $r=.65$; complexity by pleasingness, $r=-.58$ ).

An analysis of variance of symmetry and complexity and response scale reveals all main effects and interactions to be significant beyond the .001 level (response scale: $F=66.09$, $\mathrm{df}=2 / 345$; symmetry: $F=45.92, \mathrm{df}=$ 4/13455; complexity: $F=661, \mathrm{df}=7 / 13455 ; \mathrm{R}$ by $\mathrm{S}$ : $F=77.05 ; R$ by $C: F=215.28 ; S$ by $C: F=26.60 ; R$ by $S$ by $\mathrm{C}: \mathrm{F}=4.47$ ).

A comparison of the shapes of the distributions of the symmetrical vs asymmetrical figures shows no differences among the symmetrical sets but a difference between the asymmetrical and the means of the symmetrical sets. Contrasts for all three response scales in a response and symmetry interaction were significant at the .001 level (complexity, $t=15.22$; interestingness, $\mathrm{t}=4.27$; pleasingness, $\mathrm{t}=18.73$ ).

\section{Discussion}

The data indicate that symmetry affects all three response indices significantly. Symmetrical figures are rated as less complex, but more interesting and much more pleasing than their corresponding asymmetrical polygon.

Since complexity accounts for only $66 \%$ of the subjective ratings of complexity, $1 / 3$ of the variability may be due to unexplained variables and idiosyncratic characteristics of the polygons. Assuming the same variables are operating in the other response indices and only $33 \%$ of the variability in pleasingness $(43 \%$ in interestingness) is due to complexity, then approximately $1 / 3$ of the variability (about $1 / 4$ in interestingness) must be accounted for by some factor. This factor may indeed be the symmetry/asymmetry factor.

No differences between horizontal and vertical symmetry were found in the present study. Yet one would speculate that since vertical symmetry is much more common in our environment it should lead to responses of greater affect.

It appears then, that interest is directly related to complexity (up to some optimal complexity level) and is higher for symmetrical stimuli, while pleasure is inversely related to complexity but is also higher for symmetrical stimuli.

\section{References}

ATTNEAVE, F. Symmetry, information, and memory for patterns. Amer. J. Psychol. 1955, 68, 209-222.

BARRON, F. Discovering the creative personality. College Admissions 10: The behavioral sciences and education. 1963.

BARRON, F., \& WELSH, G. S. Perception as a possible factor in personality style: Its measurement by a figure preference test. J. Psychol., 1952, 33, 199-207.

BERLYNE, D. E. The influence of complexity and novelty in visual figures on orienting responses. $J$. exp. Psychol., 1958, 55, 289-296.

BERLYNE, D. E. Complexity and incongruity variables as determinants of exploratory choice and evaluative ratings. Can. J. Psychol, $1963,17,274290$.

DAY, H. Evaluations of subjective complexity, pleasingness and interestingness for a series of random polygons varying in complexity. Percept. \& Psychophys., 1967, 2, 281-286.

EISENMAN, R., \& RAPPAPORT, JOAN. Complexity preference and semantic differential ratings of complexity-simplicity and symmetryasymmetry. Psychon. Sci., 1967, 7, 147-148.

MUNSINGER, H., \& KESSEN, W. Uncertainty, structure, and preference. Psychol. Monogr., 1964, 78, No. 9 (Whole No. 586).

THOMAS, H. Preferences for random shapes: Ages six through nineteen years. Child Developm., 1966, 37, 843-859. 